\title{
One pot multi component synthetic approach towards the formation of 1,2,4-triazolo[1,5- a]pyrimidine analogues and their biological evaluation
}

\author{
Hasmukh R. Khunt, Piyush P. Pipaliya, Satish M. Ghelani, Jayesh S. Babariya, \\ Yogesh T. Naliapara* \\ Department of Chemistry, Saurashtra University, Rajkot, Gujarat, India \\ *E-mail address: naliaparachem@yahoo.co.in
}

\begin{abstract}
A library of functionalised 1,2,3-triazolo[1,5-a]pryrimidine derivatives has been synthesized by one pot multicomponenet reaction. All synthesized novel compounds were characterised by ${ }^{1} \mathrm{H}$ NMR, IR, mass and elemental analysis. Compounds were also screened against bacterial and fungle strain.
\end{abstract}

Keywords: Triazolopyrimidine, One-pot multicomponent

\section{INTRODUCTION}

Fused heteroaromatic systems are often of much greater interest than the constituent monocyclic compounds. Recently, 1,2,4-triazolo[1,5-a]pyrimidines have aroused increasing attention from the chemical and biological view points, due to their diverse pharmacological activities, such as antitumor potency [1,2] inhibition of KDR kinase [3], antifungal effect [4] and macrophage activation [5]. They have proved to be promising anticancer agents with dual mechanisms of tubulin polymerization promotion [1,2] as well as cyclin dependent kinases [2] inhibition [6].

One of the synthetic pathways to 1,2,4-triazolo[1,5-a]pyrimidines is based on the Biginelli like cyclocondensation of aromatic aldehydes and acetoacetic acid derivatives with aminoazoles containing a guanidine fragment. There are literary data about the synthesis of triazolopyrimidines by treatment of 5-amino-1,2,4-triazole or 5-aminotetrazole with aldehydes and ethyl acetoacetate or cyclic $\beta$-diketones [7-11]. The cyclocondensations were realized by heating of the starting materials in ethanol with catalytic amounts of hydrochloric acid under reflux conditions [7-9] or using DMF as solvent [10-11]. The use of acetoacetamides in these or similar reactions has not been described. However, the existing methods are suffered with some drawbacks, such as yield, time, product isolation. 
Recognizing these facts, we have synthesised a new series of $\mathrm{N}$ cyclohexyl-4,7-dihydro-5-isopropyl-7-aryl-[1,2,4]triazolo[1,5-a]pyrimidine-6-carboxamide starting from, $N$-cyclohexyl-4-methyl-3-oxo-pentanamide. The newly synthesized compounds were characterized by IR, Mass, ${ }^{1} \mathrm{H}$ NMR, ${ }^{13} \mathrm{C}$ NMR spectroscopy and elemental analysis. All the synthesized compounds were evaluated for their antimicrobial activity [12-15].

\section{RESULT AND DISCUSSION}

Initially, the reaction of $N$-cyclohexyl-4-methyl-3-oxo-pentanamide (1) with appropriate aldehyde (2) and aminoazole (3) was refluxed in 0.4 $\mathrm{mL}$ of DMF for 12-15 min. After cooling, methanol $(\sim 10 \mathrm{~mL})$ was added. The reaction mixture was allowed to stand overnight and then filtered to give the solid (Scheme 1) affords the $N$-cyclohexyl-4,7-dihydro-5isopropyl-7-phenyl-[1,2,4]triazolo[1,5- $a$ ]pyrimidine-6-carboxamide derivatives (5a-o) was obtained in excellent yield.<smiles>CC(C)C(=O)CC(=O)NC1CCCCC1</smiles>

(1)<smiles>[R]c1ccccc1C=O</smiles>

(2)<smiles>Nc1ncn[nH]1</smiles>

(3)<smiles>[R]c1ccccc1C1C(C(=O)NC2CCCCC2)=C(C(C)C)Nc2ncnn21</smiles>

(5a-0)

Scheme 1. Synthesis of pyrazolopyrimidine.

Table 1. Different Aldehydes used for the synthesis of pyrazolopyrimidines

\begin{tabular}{cccc}
\hline Entry & $\mathbf{R}$ & Yield \% & M.P. \\
\hline $5 \mathrm{a}$ & $4-\mathrm{CH}_{3} \mathrm{C}_{6} \mathrm{H}_{4}$ & 92 & $252-254$ \\
\hline $5 \mathrm{~b}$ & $4-\mathrm{ClC}_{6} \mathrm{H}_{5}$ & 91 & $260-263$ \\
\hline $5 \mathrm{c}$ & $4-\mathrm{FC}_{6} \mathrm{H}_{5}$ & 84 & $248-250$ \\
\hline $5 \mathrm{~d}$ & $4-\mathrm{OCH}_{3} \mathrm{C}_{6} \mathrm{H}_{5}$ & 90 & $245-247$ \\
\hline $5 \mathrm{e}$ & $3-\mathrm{BrC}_{6} \mathrm{H}_{5}$ & 86 & $255-257$ \\
\hline $5 \mathrm{f}$ & $3,4-\mathrm{di}^{-} \mathrm{OCH}_{3} \mathrm{C}_{6} \mathrm{H}_{3}$ & 92 & $259-261$ \\
\hline $5 \mathrm{~g}$ & $3-\mathrm{ClC}_{6} \mathrm{H}_{4}$ & 90 & $265-267$ \\
\hline $5 \mathrm{~h}$ & $\mathrm{C}_{6} \mathrm{H}_{5}$ & 86 & $245-247$
\end{tabular}




\begin{tabular}{cccc}
\hline $5 \mathrm{i}$ & $4-\mathrm{OHC}_{6} \mathrm{H}_{5}$ & 93 & $242-244$ \\
\hline $5 \mathrm{j}$ & $2-\mathrm{ClC}_{6} \mathrm{H}_{5}$ & 91 & $235-237$ \\
\hline $5 \mathrm{k}$ & $2-\mathrm{CH}_{3} \mathrm{C}_{6} \mathrm{H}_{5}$ & 88 & $255-257$ \\
\hline 51 & $2-\mathrm{OHC}_{6} \mathrm{H}_{5}$ & 92 & $257-259$ \\
\hline $5 \mathrm{~m}$ & $3-\mathrm{NO}_{2} \mathrm{C}_{6} \mathrm{H}_{5}$ & 90 & $260-262$ \\
\hline $5 \mathrm{n}$ & $4-\mathrm{NO}_{2} \mathrm{C}_{6} \mathrm{H}_{5}$ & 87 & $262-264$ \\
\hline $5 \mathrm{o}$ & $3,5-\mathrm{di}^{-}-\mathrm{OCH}_{3} \mathrm{C}_{6} \mathrm{H}_{3}$ & 85 & $256-258$ \\
\hline
\end{tabular}

The structures of (5a-o) were established on the basis of their elemental analysis and spectral data (MS, IR, and ${ }^{1} \mathrm{H}$ NMR). The analytical data for $\mathbf{5 a}$ revealed a molecular formula $\mathrm{C}_{22} \mathrm{H}_{29} \mathrm{~N}_{5} \mathrm{O}\left(\mathrm{m} / z\right.$ 379). The ${ }^{1} \mathrm{H}$ NMR spectrum revealed a doublet at $\delta=0.95-1.10 \mathrm{ppm}$ assigned to isopropyl- $\mathrm{CH}_{3}$, a multiplet at $\delta=1.17-1.58 \mathrm{ppm}$ assigned to the $-\left(5 \mathrm{x} \mathrm{CH}_{2}\right)$ protons, a singlet at $\delta=2.23 \mathrm{ppm}$ assigned to the $-\mathrm{CH}_{3}$ a multiplet at $\delta=3.18-3.28 \mathrm{ppm}$ assigned to the isopropyl- $\mathrm{CH}$ protons, a doublet at $\delta=3.34-3.40 \mathrm{ppm}$ assigned to the $-\mathrm{CH}$ protons, a singlet at $\delta=6.25 \mathrm{ppm}$ assigned to the $-\mathrm{CH}$ protons, a multiplet at $\delta=6.98-7.10$ ppm assigned to the aromatic protons, a singlet at $\delta=7.57 \mathrm{ppm}$ assigned to the $-\mathrm{CH}$ protons of triazoloring, a singlet at $\delta=7.72-7.75 \mathrm{ppm}$ assigned to $-\mathrm{NH}$ protons, a singlet at $\delta=9.66$ ppm assigned to $-\mathrm{CONH}$ protons.

\section{EXPERIMENTAL}

Thin-layer chromatography was accomplished on $0.2 \mathrm{~mm}$ precoated plates of silica gel G60 $F_{254}$ (Merck). Visualization was made with UV light $(254$ and $365 \mathrm{~nm}$ ) or with an iodine vapor. IR spectra were recorded on a FTIR-8400 spectrophotometer using DRS prob. ${ }^{1} \mathrm{H}(400$ $\mathrm{MHz})$ and ${ }^{13} \mathrm{C}(100 \mathrm{MHz}) \mathrm{NMR}$ spectra were recorded on a Bruker AVANCE II spectrometer in $\mathrm{CDCl}_{3}$. Chemical shifts are expressed in $\delta \mathrm{ppm}$ downfield from TMS as an internal standard. Mass spectra were determined using direct inlet probe on a GCMS-QP 2010 mass spectrometer (Shimadzu). Solvents were evaporated with a BUCHI rotary evaporator. Melting points were measured in open capillaries and are uncorrected.

\section{General procedure for the synthesis of substituted Triazolopyrimidines 5a-o}

A mixture of the aminoazole $(0.01 \mathrm{~mol}), \mathrm{N}$-cyclohexyl-4-methyl-3-oxo-pentanamide $(0.01 \mathrm{~mol})$ and an appropriate aromatic aldehyde $(0.01 \mathrm{~mol})$ was refluxed in $0.4 \mathrm{~mL}$ of DMF for 12-15 min. After cooling, methanol $(\sim 10 \mathrm{~mL})$ was added. The reaction mixture was allowed to stand overnight and then filtered to give the solid triazolopyrimidine products 5ao, which were crystallized from ethanol and subsequently dried in air.

\section{1. Spectroscopic data}

$N$-cyclohexyl-4,7-dihydro-5-isopropyl-7-p-tolyl-[1,2,4]triazolo[1,5-a]pyrimidine-6-

carboxamide (PVP-5a): white solid; $R_{f} 0.64$ (9:1 Chloroform : Methanol); IR (KBr): 3327, 
3093, 2939, 1648, 1586, 1492, 1261, $1069 \mathrm{~cm}^{-1} ;{ }^{1} \mathrm{H}$ NMR: $\delta 0.95-1.10\left(\mathrm{~d}, 6 \mathrm{H}, 2 \mathrm{x}{ }^{\mathrm{i}} \mathrm{prCH}_{3}\right)$, 1.17-1.58 (m, 10H, $\left.5 \times \mathrm{CH}_{2}\right), 2.23\left(\mathrm{~s}, 1 \mathrm{H}, \mathrm{CH}_{3}\right) 3.18-3.28\left(\mathrm{~m}, 1 \mathrm{H},{ }_{\mathrm{i}}^{\mathrm{i}} \mathrm{prH}\right), 3.34-3.40(\mathrm{~s}, 1 \mathrm{H}$, $\mathrm{CH}), \quad 6.25(\mathrm{~s}, 1 \mathrm{H}, \mathrm{CH}), 6.98-7.01(\mathrm{~d}, 2 \mathrm{H}, \mathrm{Ar}-\mathrm{H})$ 7.07-7.10 (d, 2H, Ar-H), $7.57(\mathrm{~s}, 1 \mathrm{H}, \mathrm{CH})$ $7.72-7.75(\mathrm{~s}, 1 \mathrm{H}, \mathrm{NH}) 9.66(\mathrm{~s}, 1 \mathrm{H}, \mathrm{CONH}) ;{ }^{13} \mathrm{C}$ NMR: $\delta 19.49,19.74,20.69,24.60,28.44$, 30.52, 32.19, 47.51, 60.49, 102.81, 126.92, 128.64, 137.01, 137.63, 141.85, 148.42, 149.30, 165.30; MS (m/z): $379\left(\mathrm{M}^{+}\right)$; Anal. Calcd for $\mathrm{C}_{22} \mathrm{H}_{29} \mathrm{~N}_{5} \mathrm{O}: \mathrm{C}, 69.63 ; \mathrm{H}, 7.70 ; \mathrm{N}, 18.45$; Found: C, 69.58; H, 7.65; N, 18.52 .

$N$-cyclohexyl-7-(4-fluorophenyl)-4,7-dihydro-5-isopropyl-[1,2,4]triazolo[1,5-

a]pyrimidine-6-carboxamide (PVP-5c): white solid; $R_{f} 0.60$ (9:1 Chloroform : Methanol); IR (KBr): 3300, 3215, 3093, 3051, 2933, 2674, 1662, 1593, 1437, 1297, $1076 \mathrm{~cm}^{-1}$; ${ }^{1} \mathrm{H}$ NMR: $\delta$ 1.009-1.114 (d, 6H, $\left.2 \times{ }_{\mathrm{i}} \mathrm{prCH}_{3}\right), 1.19-1.56\left(\mathrm{~m}, 10 \mathrm{H}, 5 \times \mathrm{CH}_{2}\right), 3.17-3.26\left(\mathrm{~m}, 1 \mathrm{H},{ }_{\mathrm{i}} \mathrm{prCH}\right)$, 3.31-3.42 (m, 1H, CH), $6.31(\mathrm{~s}, 1 \mathrm{H}, \mathrm{CH}), 7.09-721(\mathrm{~m}, 4 \mathrm{H}, \mathrm{Ar}-\mathrm{H}), 7.59$ (s, 1H, CH), 7.70$7.73(\mathrm{~s}, 1 \mathrm{H}, \mathrm{NH}), 9.73(\mathrm{~s}, 1 \mathrm{H}, \mathrm{CONH}) ; \mathrm{MS}(\mathrm{m} / \mathrm{z}): 383\left(\mathrm{M}^{+}\right)$; Anal. Calcd for $\mathrm{C}_{21} \mathrm{H}_{26} \mathrm{FN}_{5} \mathrm{O}: \mathrm{C}$, 65.78; H, 6.83; N, 18.26; Found: C, 65.68; H, 6.75; N, 18.32 .

\section{ANTIMICROBIAL SCREENING OF SYNTHESIZED PYRIMIDINES}

All the synthesized compounds were tested against different bacterial and fungal strains i.e. Pseudomonas aeruginosa, Proteus vulgaris, Escherichia Coli, Staphylococcus aureus, Candida albican for their in vitro antibacterial activity.Well Diffusion/Agar Cup Method was used and results are listed in Table 2.

Table 2. Antimicrobial Sensitivity Assay (Concentration 250/500/ $1000 \mu \mathrm{G} / \mathrm{mL}$ ).

\begin{tabular}{|l|c|c|c|c|c|c|c|c|c|c|c|c|c|c|c|c|}
\hline $\begin{array}{l}\text { Sr. } \\
\text { No. }\end{array}$ & $\begin{array}{c}\text { CODE } \\
\text { No. }\end{array}$ & \multicolumn{3}{|c|}{$\begin{array}{c}\text { Pseudomonas } \\
\text { aeruginosa }\end{array}$} & \multicolumn{3}{|c|}{$\begin{array}{c}\text { Proteus } \\
\text { vulgaris }\end{array}$} & \multicolumn{3}{|c|}{$\begin{array}{c}\text { Escherichia } \\
\text { coli }\end{array}$} & \multicolumn{3}{|c|}{$\begin{array}{c}\text { Staphylococcu } \\
\text { saureus }\end{array}$} & \multicolumn{3}{|c|}{$\begin{array}{c}\text { Candida } \\
\text { albicans }\end{array}$} \\
\hline & & $\mathbf{2 5 0}$ & $\mathbf{5 0 0}$ & $\mathbf{1 0 0 0}$ & $\mathbf{2 5 0}$ & $\mathbf{5 0 0}$ & $\mathbf{1 0 0 0}$ & $\mathbf{2 5 0}$ & $\mathbf{5 0 0}$ & $\mathbf{1 0 0 0}$ & $\mathbf{2 5 0}$ & $\mathbf{5 0 0}$ & $\mathbf{1 0 0 0}$ & $\mathbf{2 5 0}$ & $\mathbf{5 0 0}$ & $\mathbf{1 0 0 0}$ \\
\hline $\mathbf{1 .}$ & $\mathbf{5 a}$ & 1.2 & 1.4 & 2 & 1.1 & 1.3 & 1.6 & $\mathrm{R}$ & $\mathrm{R}$ & $\mathrm{R}$ & $\mathrm{R}$ & 1 & 1.2 & $\mathrm{R}$ & 1.2 & 1.5 \\
\hline $\mathbf{2 .}$ & $\mathbf{5 b}$ & 1.2 & 1.3 & 1.7 & 1.1 & 1.4 & 1.6 & $\mathrm{R}$ & $\mathrm{R}$ & $\mathrm{R}$ & 1.2 & 1.3 & 1.6 & 1 & 1.3 & 1.8 \\
\hline $\mathbf{3 .}$ & $\mathbf{5 c}$ & 1.5 & 1.3 & 1.5 & $\mathrm{R}$ & 1.1 & 1.4 & 1.1 & 1.2 & 1.3 & $\mathrm{R}$ & 1 & 1.2 & 1.1 & 1.5 & 2 \\
\hline $\mathbf{4 .}$ & $\mathbf{5 d}$ & 1.6 & 1.2 & 1.4 & 1 & 1.3 & 1.6 & $\mathrm{R}$ & $\mathrm{R}$ & $\mathrm{R}$ & 1.3 & 1.4 & 1.6 & 1.1 & 1.4 & 1.8 \\
\hline $\mathbf{5 .}$ & $\mathbf{5 e}$ & 1.4 & 1.3 & 1.6 & $\mathrm{R}$ & 1.2 & 1.4 & $\mathrm{R}$ & $\mathrm{R}$ & $\mathrm{R}$ & 1.2 & 1.4 & 1.6 & 1 & 1.3 & 1.7 \\
\hline $\mathbf{6 .}$ & $\mathbf{5 f}$ & 1.3 & 1.5 & 1.9 & 1 & 1.2 & 1.3 & 1.3 & 1.4 & 1.7 & 1.1 & 1.4 & 1.5 & 1.1 & 1.4 & 1.8 \\
\hline $\mathbf{7 .}$ & $\mathbf{5 g}$ & 1.9 & 1.5 & 1.8 & 1.1 & 1.4 & 1.7 & 1.2 & 1.4 & 1.8 & 1.4 & 1.5 & 2 & 1.2 & 1.4 & 1.7 \\
\hline $\mathbf{8 .}$ & $\mathbf{5 h}$ & 1.4 & 1.7 & 2 & 1.1 & 1.3 & 1.5 & 1.1 & 1.1 & 1.3 & 1.4 & 1.6 & 2 & 1.1 & 1.3 & 1.5 \\
\hline $\mathbf{9 .}$ & $\mathbf{5 i}$ & 1.2 & 1.3 & 1.5 & $\mathrm{R}$ & $\mathrm{R}$ & $\mathrm{R}$ & $\mathrm{R}$ & $\mathrm{R}$ & $\mathrm{R}$ & 1.3 & 1.4 & 1.7 & $\mathrm{R}$ & 1.3 & 1.7 \\
\hline $\mathbf{1 0}$ & $\mathbf{5 j}$ & 1.7 & 1.9 & 2 & 1.8 & 1.8 & 2 & 1.1 & 1.8 & 1.8 & 1.5 & 1.7 & 1.9 & 1.8 & 1.8 & 2 \\
\hline
\end{tabular}




\begin{tabular}{|c|c|c|c|c|c|c|c|c|c|c|c|c|c|c|c|c|}
\hline 11. & $5 k$ & 1.1 & 1.2 & 1.3 & $\mathrm{R}$ & 1 & 1.2 & 1.1 & 1.2 & 1.4 & 1.1 & 1.2 & 1.5 & 1.1 & 1.5 & 1.9 \\
\hline 12. & 51 & 1.3 & 1.4 & 1.9 & 1.3 & 1.7 & 2.1 & 1.2 & 1.5 & 2 & 1.1 & 1.5 & 1.9 & 1.1 & 1.4 & 1.6 \\
\hline 13. & $5 \mathrm{~m}$ & 1.2 & 2 & 1.5 & 1.1 & 1.4 & 1.9 & 1.3 & 1.4 & 1.9 & 1.2 & 1.6 & 2 & 1.2 & 1.5 & 2 \\
\hline 14. & $5 n$ & $\mathrm{R}$ & $\mathrm{R}$ & $\mathrm{R}$ & 1.1 & 1.3 & 1.7 & 1.1 & 1.3 & 1.6 & $\mathrm{R}$ & $\mathrm{R}$ & $\mathrm{R}$ & 1.1 & 1.4 & 1.8 \\
\hline 15. & 50 & 1.4 & 1.6 & 2 & 1 & 1.2 & 1.4 & $\mathrm{R}$ & $\mathrm{R}$ & $\mathrm{R}$ & 1.1 & 1.2 & 1.5 & 1.2 & 1.5 & 2 \\
\hline 16. & $\mathbf{A}$ & \multicolumn{3}{|c|}{1.8} & \multicolumn{3}{|c|}{1.8} & \multicolumn{3}{|c|}{1.9} & \multicolumn{3}{|c|}{1.9} & \multicolumn{3}{|c|}{-} \\
\hline 17. & CPD & \multicolumn{3}{|c|}{2.2} & \multicolumn{3}{|c|}{2.1} & \multicolumn{3}{|c|}{2.1} & \multicolumn{3}{|c|}{2.2} & \multicolumn{3}{|c|}{-} \\
\hline 18. & GF & \multicolumn{3}{|c|}{1.8} & \multicolumn{3}{|c|}{1.9} & \multicolumn{3}{|c|}{2.0} & \multicolumn{3}{|c|}{2.0} & \multicolumn{3}{|c|}{ - } \\
\hline 19. & GRF & \multicolumn{3}{|c|}{-} & \multicolumn{3}{|c|}{-} & \multicolumn{3}{|c|}{-} & \multicolumn{3}{|c|}{-} & \multicolumn{3}{|c|}{2.6} \\
\hline 20. & FLC & \multicolumn{3}{|c|}{-} & \multicolumn{3}{|c|}{-} & \multicolumn{3}{|c|}{-} & \multicolumn{3}{|c|}{-} & \multicolumn{3}{|c|}{2.8} \\
\hline
\end{tabular}

Note: Zone of inhibition interpretation is as follows.

1. ZONE SIZE $<1.0$ C.M. - RESISTENT(R)

2. ZONE SIZE 1.0 To 1.5 - INTERMEDIATE

3. ZONE SIZE $>1.5-$ SENSITIVE

STD Antibiotic Sensitivity Assay Concentration $40 \mu \mathrm{G} / \mathrm{ml}$

A: AMPICILLIN

CPD: $\quad$ CEFPODOXIME

GF: GATIFLOXACIN

GRF: GRESIOFULVIN

FLC: FLUCONAZOLE

\section{CONCLUSIONS}

In summary, we have described the synthesis of 1,2,4-triazolo[1,5-a]pyrimidines derivatives in excellent yields. The reaction of $N$-cyclohexyl-4-methyl-3-oxo-pentanamide (1) with appropriate aldehyde (2) and aminoazole (3) was refluxed in DMF affords the $\mathrm{N}$ cyclohexyl-4,7-dihydro-5-isopropyl-7-phenyl-[1,2,4]triazolo[1,5- $a]$ pyrimidine-6-carboxamide derivatives was obtained in excellent yield. All the synthesized compounds are evaluated for their antimicrobial activity. The investigation of antibacterial and antifungal screening data revealed that all the tested compounds 5a-o showed moderate to potent activity. The compounds $\mathbf{5 j}$ and $\mathbf{5 l}$ showed comparatively good activity against all the bacterial strains.

\section{ACKNOWLEDGEMENT}

Author thankful to Department of Chemistry, Saurashtra University for providing facilities and SAIF, Punjab University for instrumental support. 


\section{References}

[1] N. Zhang, A. Semiramis, N. Thai, J. Med. Chem. 50 (2007) 319.

[2] L. Havlicek, K. Fuksova, V. Krystof, Bioorg. Med. Chem. 13 (2005) 5399.

[3] M. Fraley, W. Hoffman, R. Rubino, Bioorg. Med. Chem. Lett. 12 (2002) 2767.

[4] Q. Chen, X. Zhu, Z. Liu, Eur. J. Med. Chem. 43 (2008) 595.

[5] S. Uryu, S. Tokuhiro; T. Murasugi, Brain Research 946 (2002) 298.

[6] L. Havlicek; K. Fuksova, V. Krystof, Bioorg. Med. Chem. 13 (2005) 5399

[7] I. Drizin, M. Holladay, L. Yi, H. Zhangm, S. Gopalakrishnan, M. Gopalakrishnan, K. Whiteaker, S. Buckner, J. Sullivan, W. Carrol, Bioorg. Med. Chem. Lett. 12 (2002) 1481.

[8] O. Fedorova, M. Zhidovinova, G. Rusinov, I. Ovchinnikova, IzV. Akad. Nauk. Ser. Khim (2003) 1677.

[9] M. Pryadeina, Y. Burgart, V. Saloutin, M. Kodess, E. Ulomsky, V. Rusinov, Zh. Org. Khim. 40 (2004) 938.

[10] V. Lipson, S. Desenko, M. Shirobokova, Borodina, Chem. Heterocycl. Compd. 39 (2003) 1213.

[11] V. Lipson, S. Desenko, S. Shishkina, M. Shirobokova, O. Shishkin. V. Orlov, Khim. Geterotsikl. Soedin. 39 (2003) 1194.

[12] G. Thirunarayanan, International Letters of Chemistry, Physics and Astronomy 5 (2014) 89-98.

[13] Nirav M. Shah, Hitendra S. Joshi, International Letters of Chemistry, Physics and Astronomy 6 (2014) 56-60.

[14] Piyush B. Vekariya, Jalpa R. Pandya, Vaishali Goswami, Hitendra S. Joshi, International Letters of Chemistry, Physics and Astronomy 7 (2014) 45-52.

[15] Sagar P. Gami, Kalpesh V. Vilapara, Hasmukh R. Khunt, Jayesh S. Babariya, Yogesh T. Naliapara, International Letters of Chemistry, Physics and Astronomy 11(2) (2014) 127-134. 